\title{
NOUVELLE
}

\section{Protéolyse membranaire : le clivage de ErbB-4 permet sa migration vers le noyau}

Bernadette Allinquant
Cnrs UMR 8542, દ́cole

Normale Supérieure,

46 , rue d'Ulm,

75230 Paris Cedex 05,

France. domaines transmembranaires, probablement multifonctionnelles, associées à une activité aspartyl protéase [7]. Leur activité protéolytique directe, ou leur participation à la protéolyse comme co-facteur d'une $\gamma$ sécrétase, est à discuter. L'implication des présénilines dans le clivage intramembranaire a été démontrée par l'invalidation du gène $P S 1$, qui bloque l'activité $\gamma$-sécrétase sans modifier le clivage au site $\beta$. De plus, les souris dont le gène PSI a été invalidé présentent un phénotype létal embryonnaire semblable à celui des souris dont le récepteur Notch est inactivé. Cette inhibition de la voie Notch en l'absence de présénilines a aussi été observée chez la drosophile et chez C. elegans $[5,7]$.

Pour Notch comme pour ErbB-4, le clivage intramembranaire par une $\gamma$ sécrétase ne survient qu'après un premier clivage extracellulaire, à proximité de la membrane, par une métalloprotéase (TACE) (FigureI). Il est intéressant de constater qu'il en est de même pour I'APP dont le clivage intramembranaire n'a lieu qu'une fois effectué le clivage en $\beta$ par une aspartyl protéase (BACE) ( $\beta$-site APP cleaving enzyme) $[4,7]$. Notons que, dans sa voie non amylö̈dogénique, I'APP peut aussi être clivé au site $\alpha$ par deux métalloprotéases, ADAMIO (a disintegrin and a metalloprotease) et TACE (Figure 1). Ce dernier clivage est suivi d'un clivage en $\gamma$ donnant ainsi naissance au peptide p3 (Figure 1). Dans tous les cas, le clivage par une $\gamma$-sécrétase pose la question du compartiment cellulaire impliqué, les présénilines étant majoritairement présentes au niveau du réticulum endoplasmique et du Golgi [7] $(\rightarrow)$. Les mécanismes de protéolyse contrôlée intra- $(\rightarrow) \mathrm{m} / \mathrm{s}$ 2002, $\mathrm{n}^{\circ} 2$, p. 157
1. Les $\gamma$-sécrétases sont des enzymes (encore non clairement identifiées) qui clivent $\mathrm{ErB}-4$ dans le plan de la membrane. Les présénilines (PS1 et PS2) sont associées à ces activités. mentation du taux de peptide $A \beta[7]$. Les présénilines sont des protéines à 8 
membranaire étaient déjà connus pour des systèmes qui règlent la différenciation cellulaire, le métabolisme lipidique ou la réponse aux protéines chaperons. Dans tous les cas connus, le clivage intramembranaire suit celui du domaine $\mathrm{N}$-terminal. Un des exemples est le SREBP (sterol regularory element binding protein), protéine de type 2 (extrémités $\mathrm{N}$ - et $\mathrm{C}$-terminales dirigées vers le cytoplasme) qui s'associe à la protéine SCAP (SERBP cleavage-activating protein). En fonction du taux intracellulaire de stérol, SCAP adresse SREBP du réticulum endoplasmique au Golgi cis-médian où prennent place les deux protéolyses successives. Le frag- ment de SREBP ainsi libéré gagne le noyau où il active la transcription des enzymes contrôlant l'homéostasie du cholestérol. La protéolyse intramembranaire de SREBP ne requiert pas les présénilines, contrairement à celle de Irel, protéine impliquée dans la réponse aux protéines mal repliées présentes dans le réticulum endoplasmique [8].

Dans tous les exemples connus de protéolyse intramembranaire, qu'elles fassent ou non intervenir des présénilines, le domaine cytoplasmique libéré par ce clivage se détache de la membrane et migre dans le noyau. Dans le cas de I'APP, cette migration nécessite une

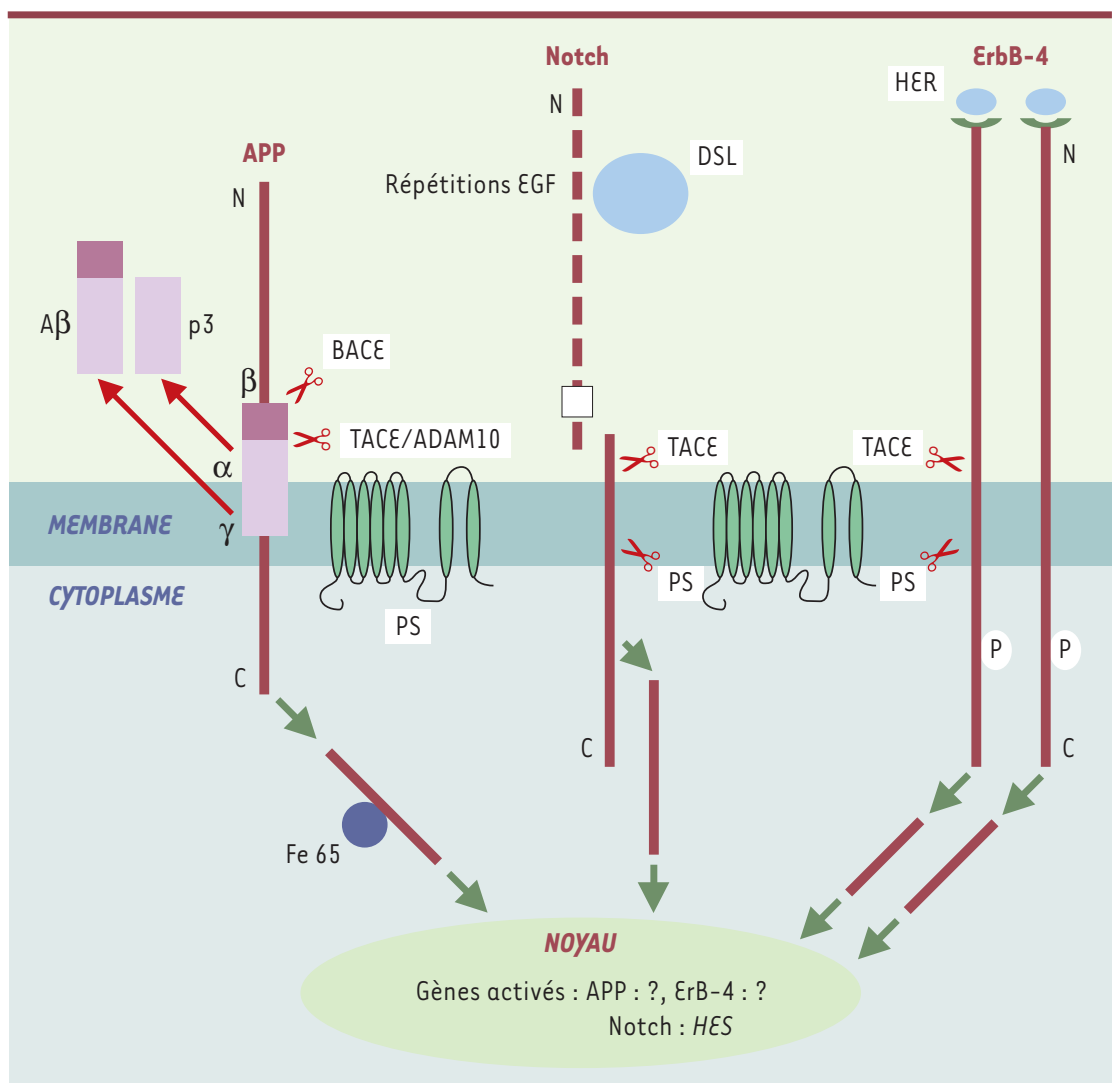

Figure 1. APP, Notch et $\varepsilon r b B-4$ : analogies dans le mécanisme de signalisation. Ces trois protéines transmembranaires vont sous l'action d'un ligand - DSL (Delta, Serrate, Lag2) pour Notch, et pour HER (heréguline) $\varepsilon r b B-4$ - subir un premier clivage par une métalloprotéase TACE suivi d'un second, cette fois intramembranaire par une $\gamma$-sécrétase, associée aux présénilines (PS). Pour l'APP, la relation entre clivages et ligand reste à définir. Dans tous les cas, ces clivages libèrent un fragment cytoplasmique qui est adressé au noyau. Dans le cas de Notch, après migration nucléaire, le domaine cytoplasmique active les gènes de la famille HES (hairy/enhancer of split).

interaction avec la protéine Fe65 [9]. Les gènes activés dans le noyau par le domaine cytoplasmique restent à définir pour l'APP et ErbB-4. Dans le cas de Notch, les gènes de la famille HES (hairy/enhancer of split) ont été identifiés.

Le clivage intramembranaire d' $\varepsilon r b B-4$ et de Notch suit l'interaction d'un ligand avec la partie extracellulaire de ces récepteurs. Les ligands de l'hétérodimère Notch conduisant à cette cascade d'événements sont connus : ils font partie de la famille DSL, Delta, Serrate (jagged chez l'homme), Lag2 (drosophile). ErbB-4 n'est clivé au site intramembranaire que s'il est stimulé par la héréguline ou par le TPA (tetradecanoylphorbol acetate), activateur de la PKC qui mime l'action du ligand (Figure 1). Aucun ligand extracellulaire de I'APP n'a été clairement identifié et la relation entre processus de clivage et fixation d'un tel ligand reste à définir. Il est possible que la réponse du récepteur varie avec la nature du ligand. Ainsi, l'interaction de Notch avec Wingless, un autre ligand, entraîne l'activation d'autres gènes et implique probablement un mécanisme de signalisation différent [10]. De même, si on mime un ligand potentiel de l'APP par la fixation d'une immunoglobuline reconnaissant le domaine $\mathrm{N}$-terminal, il en résulte l'induction d'une signalisation via Go [4]. Pour tous ces récepteurs, il sera intéressant d'identifier les gènes spécifiquement activés (ou réprimés) par l'adressage nucléaire de leurs domaines cytoplasmiques, ou de fragments dérivés de ces domaines, et de les placer dans la voie de transduction du signal. Ces travaux permettront de mieux comprendre le rôle physiologique de ce mécanisme de signalisation inattendu mais qui pourrait se révéler plus général que prévu. $\diamond$

From membrane to the nucleus, clivage of $\varepsilon r b B-4$ triggers migration to the nucleus 
1. Yarden Y, Sliwkowski MK.

Untangling the ErbB signalling network. Nat Rev Mol Cell Biol 2001; 2 : 127-37.

2. Ni CY, Murphy MP, Golde DE, Carpenter $G$. $\gamma$-secretase cleavage and nuclear localization of ErbB-4 receptor tyrosine kinase. Science 2001; 294 : 2179-81.

3. Lin SY, Makino K, Xia W, et al. Nuclear localization of EGF receptor and its potential new role as a transcription factor. Nat Cell Biol $2001 ; 3$ : 802-8.

4. De Strooper B, Annaert W. Proteolytic processing and cell biological functions of the amyloid precursor protein. J Cell Sci 2000 ; 113: 1857-70.

5. Weinmaster $G$. Notch signal transduction: a real Rip and more. Curr Opin Genet Dev $2000 ; 10: 363-9$.

6. Okamoto I, Kawano $Y$, Murakami $D$, et al.
Proteolytic release of CD44 intracellular domain and its role in the CD44 signaling pathway. J Cell Biol 2000; $155: 755-62$.

7. De Strooper B, Annaert W. Presenilins and the intramembrane proteolysis: facts and fiction. Nat Cell Biol 2000 ; 3 : ع221-5.

8. Brown MS, Ye J, Rawson RB, Goldstein JL. Regulated intramembrane proteolysis: a control mechanism conserved from bacteria to humans. Cell

\section{NOUVELLE}

\section{A la recherche d'inhibiteurs de N-WASP}

Christophe Le Clainche

> Une cellule n'est jamais immobile, elle se déplace et change de forme. On sait depuis longtemps que le cytosquelette d'actine joue un rôle majeur dans ces processus dynamiques. Ce qui excite maintenant la curiosité des biologistes, c'est de comprendre comment la signalisation contrôle la dynamique du cytosquelette d'actine. Un grand pas a été franchi avec la découverte des protéines de la famille WASP (Wiskott Aldrich syndrome protein). Ces protéines fonctionnent comme des centres d'intégration vers lesquels convergent les signaux activateurs comme le phosphoinositide $\mathrm{PIP}_{2}$, les protéines Grb2 et Nck liées aux récepteurs à activité tyrosine kinase ou la petite protéine $\mathrm{G} \mathrm{Cdc42.} \mathrm{Ces}$ activateurs déplacent l'équilibre conformationnel des protéines WASP de la forme fermée inactive vers la forme ouverte active. L'ouverture de la protéine WASP démasque un domaine appelé VCA (Verproline-Cofiline-Acide), qui fixe et active le complexe Arp2/3 dont la fonction est de multiplier les filaments d'actine en créant des branches (Figure 1) (pour revue, voir [1]).

Ces récentes découvertes ont accéléré la mise au point de nouvelles approches. Alors que les techniques de vidéo-microscopie se développaient à grands pas, on ne voyait pourtant pas apparaître de nouvelles molécules adaptées à l'étude de phénomènes aussi transitoires que le remodelage du cytosquelette d'actine. Les petites molécules sont des outils précieux pour l'étude de phénomènes dynamiques dans des cellules en culture quand elles sont capables de traverser les membranes et d'agir instantanément. Depuis longtemps, des molécules comme la cytochalasine $D$, qui inhibe la polymérisation de l'actine, font merveille pour ce type d'étude. Malheureusement, ces molécules sont rares car ce sont souvent des composés naturels. Aujourd'hui, la synthèse de banques de peptides par chimie combinatoire offre un réservoir illimité de molécules dans lequel les biologistes peuvent puiser pour trouver de nouvelles drogues. C'est cette approche que le groupe de Kirschner a utilisé pour rechercher des peptides synthétiques capables d'inhiber spécifiquement l'assemblage des filaments d'actine branchés induit par la voie PIP [2]. Cette équipe a mis au point un test
$2000 ; 100: 391-8$

9. Cao X, Sudhoff TC. A transcriptively active complex of APP with Fe65 and histone acetyltransferase Tip 60. Science 2001 ; 293 : 115-20.

10. Wesley CS, Saez L. Notch responds differently to Delta and Wingless in cultured Drosophila cells. J Biol Chem 2000 ; 275 : 9099-101.
Dynamique du cytosquelette, LEBS, Cnrs UPR 9063,

Gif-sur-yvette, France.

Christophe.LeClainche@lebs.cnrs-gif.fr

dans lequel des liposomes sont incubés avec de l'actine marquée à la rhodamine et un extrait d'œuf de xénope qui contient notamment Cdc42, N-WASP et Arp2/3. Ils observent alors l'assemblage de l'actine, induit par la voie $\mathrm{PIP}_{2}$ à la surface des liposomes, grâce à la fluorescence de la rhodamine. Ce test leur a permis de cribler des banques combinatoires de peptides cycliques. Les peptides cycliques sont plus rigides que les peptides linéaires, cela augmente leur affinité pour la cible en diminuant le coût énergétique de leur fixation. Parmi les composés testés, les auteurs ont identifié un peptide appelé 187-1 qui inhibe complètement la polymérisation de l'actine induite par $\mathrm{PIP}_{2}$. L'étude approfondie du mécanisme d'inhibition montre que ce peptide se fixe à $\mathrm{N}$ WASP et stabilise sa conformation inactive (Figure 1). La conformation inactive de $\mathrm{N}$ WASP fait intervenir une interaction intramoléculaire entre deux domaines de $\mathrm{N}$ WASP pour masquer le domaine VCA qui active Arp2/3. La nature des domaines de $\mathrm{N}$-WASP qui interagissent est actuellement discutée. II a été proposé que le domaine qui fixe $\mathrm{Cdc42}$ (GBD) interagit avec le 